\title{
The fundamental basis of inflammatory bowel disease
}

\author{
Warren Strober, Ivan Fuss, and Peter Mannon
}

Mucosal Immunity Section, Laboratory of Host Defenses, National Institute of Allergy and Infectious Diseases, NIH, Bethesda, Maryland, USA.

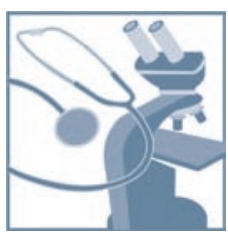

Two broad hypotheses have arisen regarding the fundamental nature of the pathogenesis of inflammatory bowel diseases (IBDs, which include ulcerative colitis and Crohn disease). The first contends that primary dysregulation of the mucosal immune system leads to excessive immunologic responses to normal microflora. The second suggests that changes in the composition of gut microflora and/or deranged epithelial barrier function elicits pathologic responses from the normal mucosal immune system. Here we examine these hypotheses and conclude that IBD is indeed characterized by an abnormal mucosal immune response but that microbial factors and epithelial cell abnormalities can facilitate this response.

The inflammatory bowel diseases (IBDs), represented mainly by ulcerative colitis and Crohn disease but also including noninfectious inflammations of the bowel, have posed an enigma to gastroenterologists and immunologists alike since their first modern descriptions some $75-100$ years ago. Powerful new investigative techniques, however, are gradually leading to an increased understanding of the major pathophysiologic processes underlying these diseases, enabling in turn the development of powerful new therapies.

Idiopathic IBDs such as Crohn disease and ulcerative colitis occur in clinically immunocompetent individuals whose characteristic symptoms and signs arise from a robust, cytokine-driven (yet noninfectious) inflammation of the gut (1). Crohn disease is associated with excess IL-12/IL-23 and IFN- $\gamma /$ IL-17 production that affects the small bowel and colon with discontinuous ulceration and full thickness bowel wall inflammation often including granulomas. Patients report gastrointestinal symptoms of abdominal pain, diarrhea, and rectal bleeding as well as systemic symptoms of weight loss, fever, and fatigue. Crohn disease patients can also develop obstructing strictures of the bowel and inflammatory connections (fistulae) between segments of bowel or between the bowel and skin and other organs. In comparison, ulcerative colitis is associated with excess IL-13 production, primarily affecting the colon, with a continuous inflammation of the mucosa nearly always involving the rectum and extending proximally (2). The symptoms are similar to Crohn disease, although fistula development does not occur. Usually both conditions are chronic and relapsing, though ulcerative colitis is curable by surgical removal of the colon (surgery for Crohn disease treats bowel blockage, fistula complications, and intractable bleeding and pain but is not used for cure) (3). Medical therapy relies on classic antiinflammatory and immunosuppressant drugs: corticosteroids, mesalamine compounds, azathioprine, and derivatives of the latter. The evidence for their utility and mechanism of action has been described elsewhere (4-6). Suffice it to say here, that these agents vary in their ability to induce and

Nonstandard abbreviations used: CARD15, caspase recruitment domain protein 15 ; ECOVA, E. coli-expressing OVA peptide; HD5, human $\alpha$-defensin 5 ; IBD, inflammatory bowel disease; MDP, muramyl dipeptide; NOD2, nucleotide-binding oligomerization domain containing 2 ; PGN, peptidoglycan.

Conflict of interest: The authors have declared that no conflict of interest exists. Citation for this article: J. Clin. Invest. 117:514-521 (2007). doi:10.1172/JCI30587. maintain control of symptoms as well as in their tolerability and toxicities. Newer biological drugs such as anti-TNF- $\alpha$ antibodies targeting the general inflammatory cytokine, TNF- $\alpha$, have added greatly to our ability to control IBD, but even this therapy is limited by lack or loss of efficacy and associated toxicities (7). Emerging therapies for IBD are focusing on major effector cytokines as they are identified in ongoing investigations $(8,9)$, for instance using an anti-IL-12p40 antibody to neutralize the effects of IL-12 and IL-23 in Crohn disease (10). Moreover, the occurrence of IBDs in immunodeficient states (such as chronic granulomatous disease [ref. 11] and common variable immunodeficiency [ref. 12]) and genetic syndromes (such as Hermansky-Pudlak syndrome [ref. 13]) and following immunotherapies such as anti-CTLA-4-blocking antibody (14) suggests that many components of the immune response have a role in IBD susceptibility.

Most students studying the pathogenesis of IBD have adopted the view that the disease is due to a dysfunctional interaction between bacterial microflora of the gut and the mucosal immune system. In one version of this view, the microflora are both qualitatively and quantitatively normal and the disease defect lies within the mucosal immune system. In this case, the normal state of immunologic tolerance to microbial antigens in the GI tract is disturbed either by the presence of a defective mucosal effector $T$ cell population that overreacts to usual microbial antigens or, alternatively, by the presence of a defective mucosal Treg cell population that underreacts to usual microbial antigens such that even normal effector $T$ cells are not properly modulated. In a second and opposing version of this view, a fundamental abnormality exists in the gut microflora, either in the number or type of organisms that comprise the population or in the extent to which the organisms confront on the mucosal immune system. This again results in a loss of tolerance, since the microflora are subsequently able to induce a normal immune system to respond excessively to microbial antigens. Here we attempt to marshal the available evidence supporting each of these theories so as to decide which of them (if not a combination of both) best explains IBD.

The case for the presence of a mucosal immune defect $I B D$ requires gut commensal organisms. Several lines of evidence support the notion that an excessive immune response to an essentially normal population of gut microflora underlies the pathogenesis 
of IBD. Some 10 years ago, studies of IBD patients conducted by Duchmann and colleagues showed that cells derived from inflamed intestinal IBD tissue exhibit robust stimulation when cultured with sonicates of autologous or heterologous gut microflora, whereas cells from normal individuals responded only to sonicates of heterologous microflora (15). These studies suggested that patients lack tolerance to the antigens present in autologous microflora. However, one could not be sure that this observation was not secondary to a disrupted epithelium that led to increased exposure to autologous microflora and thus enhanced reactivity. Similar hyperreactivity to normal gut microflora was observed in a number of animal models with vastly different underlying genetic defects (IL-2, IL-10, or $\mathrm{G}_{\mathrm{ia} 1}$ deficiency) that led to abnormal effector cell or Treg responses (16). However, gut inflammation did not occur if the mouse at risk was maintained in a strictly germ-free environment. Thus it was apparent that normal gut commensals could drive mucosal inflammation in these mouse models and, to the extent that these models mimic human IBD, they also drive mucosal inflammation in human disease. One caveat to this conclusion is that mice with gross abnormalities of gut epithelial barrier function may also develop inflammation despite the presence of a normal mucosal immune system (17).

Evidence for IBD immune defects from mouse models. A more compelling body of evidence supporting the abnormal immune system thesis involves the role of innate immune factors in the induction of mucosal inflammation in animal models. It is now well established that microbial components are necessary for the initiation of abnormal inflammatory responses because of their capacity to stimulate cells via surface membrane and endosomal TLRs, intracytoplasmic members of the nucleotide-binding oligomerization domain containing (NOD) and Pyrin families of proteins, and perhaps other molecules that recognize common bacterial, viral, and parasitic components $(18,19)$. Evidence of this effect comes from studies of STAT3-deficient mice that have defects in IL-10 function and the knowledge that STAT3 is essential for IL-10 signaling (20). These mice develop a spontaneous enterocolitis that, like human Crohn disease, is dependent on the production of IL-12p40. Interestingly, when the STAT3-deficient mice were crossed with TLR4-deficient mice to obtain STAT3/TLR4 double-deficient mice, it was found that the latter animals manifested significantly less colitis and IFN- $\gamma$ production, indicating that microflora-derived LPS (the TLR4 ligand) was necessary for the development of the inflammatory Th1-type $T$ cells. Recently, this finding was elegantly corroborated by studies of IL-10-deficient mice that lacked any kind of TLR response (as a result of the knockout of myeloid differentiation factor 88 [MyD88], an essential intracellular component of most TLR signaling pathways) which also failed to develop colitis (21). These findings suggest that one can identify genes in humans that affect innate immune responses and contribute to the development of IBD.

NOD2/caspase recruitment domain protein 15 mutations seen in buman Crobn disease. A disease susceptibility gene linked to the innate immune response has been identified. It is the caspase recruitment domain protein 15 (CARD15) gene, which encodes NOD2, and that is located in the previously defined disease susceptiptibility region at chromosome 16q12 known as inflammatory bowel disease-1 (IBD1). A mutant form of NOD2 is present in some $10 \%-20 \%$ of Caucasian patients with Crohn disease, with three specific mutations - Arg702Trp, Gly908Arg, and a frameshift deletion mutation at Leu 1007 - accounting for $82 \%$ of all Crohn-associated CARD15 mutations $(22,23)$. Patients homozygous for these mutations have a 20- to 40-fold increased risk for disease development, whereas heterozygotes have only a 2- to 4-fold increased risk. NOD2 belongs to a family of intracellular proteins possessing a NOD linked at its carboxy terminus by a leucine-rich repeat (LRR) domain (19). The LRR domain is the microbial component recognition unit and is structurally similar to the LRR recognition unit found in TLRs, whereas the NOD domain is important in the activation of the molecule following its recognition of ligand. In some members of this protein family, such as NOD1 and NOD2, the NOD domain is linked at its aminoterminal end to a CARD domain and activation of the NOD molecule leads to activation of a second molecule known as receptor interacting protein-like interacting caspase-like apoptosis regulatory protein kinase, RICK, (also known as RIP2) (24). The latter then causes downstream activation of the key proinflammatory transcription factor, NF-кB, by promoting ubiquitination of IKB kinase $\gamma(\mathrm{IKK} \gamma)(25)$. The microbial ligand recognized by NOD2 is a peptide fragment, muramyl dipeptide (MDP), derived from peptidoglycan (PGN), a component of the bacterial wall of virtually all bacteria. NOD2 is expressed in APCs and in epithelial cells at the base of small intestinal crypts known as Paneth cells. Thus, its dysfunction in either of these cell types could be involved in the pathogenesis of Crohn disease.

Initial study of NOD2 function revealed that exposure of APCs to MDP induced NOD2 to stimulate low-level activation of NF- $\mathrm{B}$ and the production of NF- $\mathrm{kB}$-dependent inflammatory factors such as IL-8 (26). Thus, it was not surprising that constructs of NOD2 mutations found in Crohn disease manifested decreased $\mathrm{NF}-\kappa \mathrm{B}$ activation and function in some in vitro studies (18). This posed a dilemma: how can one explain the occurrence of increased inflammation dependent on NF- $\mathrm{BB}$ in individuals bearing a mutation impairing NF- $\kappa \mathrm{B}$ activation? One answer was to postulate that the impaired NOD2 function led to a host defense defect that allowed increased bacterial colonization of the gut wall, and then later, in turn, led to stimulation of NF-KB via NOD2-independent mechanisms. Another hypothesis was prompted by the observation that the ligand for TLR2, PGN, the parent molecule of MDP, can activate NF-кB independently of NOD2 (27). These data showed that, quite surprisingly, APCs from NOD2-deficient mice had substantially increased IL-12 responses when stimulated with PGN as compared with NOD2-intact APCs (other TLR ligand responses were equivalent). In addition, they showed that the PGN-driven IL-12 responses of NOD2-intact APCs were inhibited by the presence of MDP, but NOD2-deficient APC responses were not. Additional data showed that in the absence of NOD2 signaling, TLR2 stimulation by PGN gave rise to increased NF- $\kappa \mathrm{B}$ activation, especially of c-Rel, an NF- $\kappa \mathrm{B}$ component critical to IL-12 transcription (19). The most reasonable interpretation of these findings was that activation of NOD2 by MDP, while it may lead to low-level NF-кB activation on its own, leads to overall downregulation of NF- $\mathrm{KB}$ by its simultaneous dampening effect on PGN-stimulated TLR2 activity. Thus, TLR2 and NOD2 function appeared to be linked via PGN, and the NOD2 mutations led to a gain-of-function abnormality and increased activation of NF-кB.

It should be noted, however, that these findings have not yet been fully corroborated by studies of actual patients with Crohn disease that have CARD15 mutations. In this regard, while several studies have shown that these patients generally display reduced cytokine responses to either MDP alone (as expected) or to TLR stimulants plus MDP, there is preliminary data showing abnormally high IL-12p70 responses (28). 

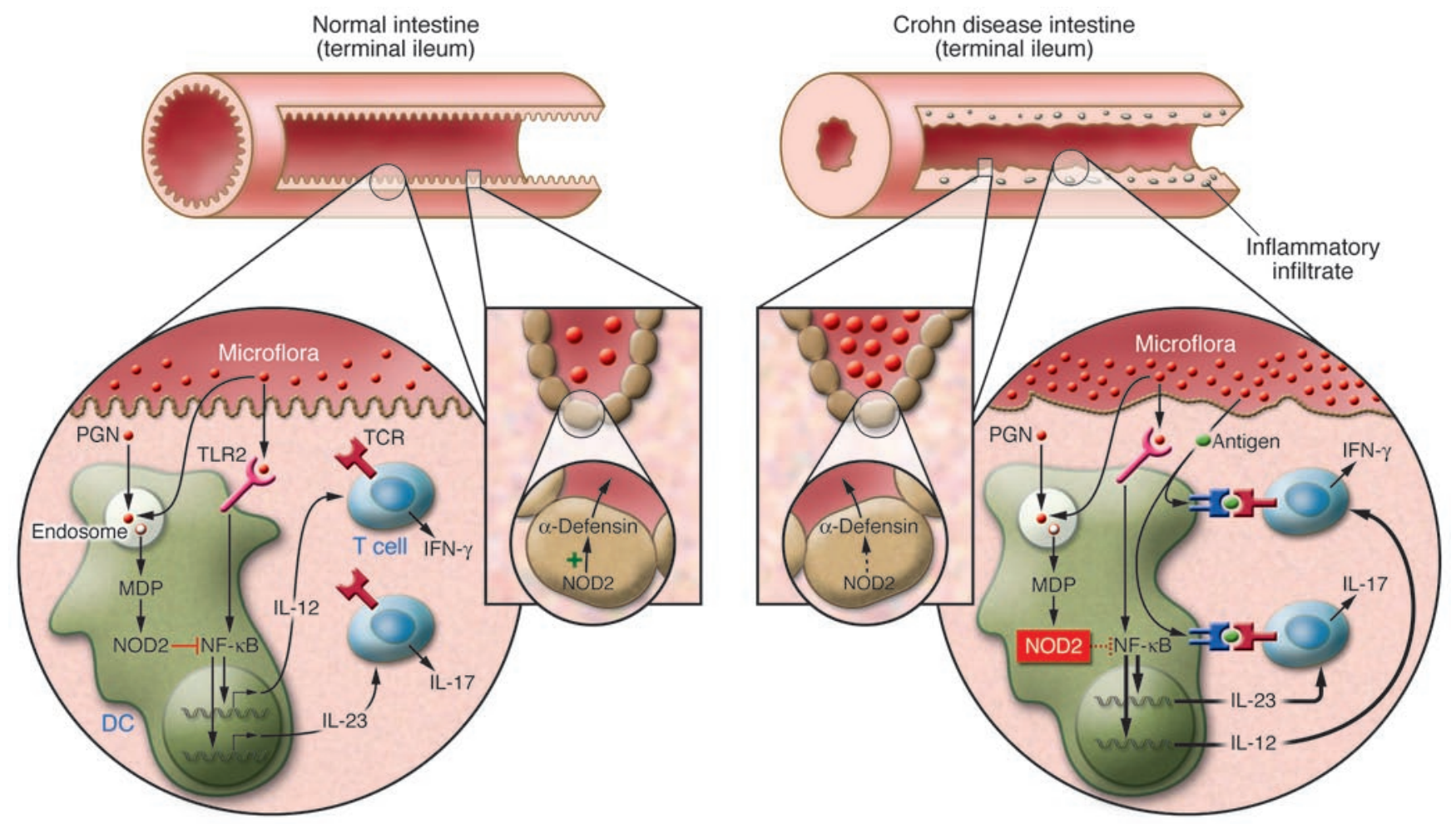

Figure 1

Critical features of the mucosal immune response of normal individuals and patients with Crohn disease with defective NOD2 function. PGN derived from the walls of commensal bacteria is sensed by TLR2 on the surface of dendritic cells in the intestinal lamina propria (left inset). This leads to TLR2 ligation and the downstream activation of NF-KB, the key transcription factor necessary for the differentiation of cells producing IFN- $\gamma$ and IL-17, the proinflammatory cytokines responsible for Crohn disease. PGN, however, is also broken down in endosomes and is thus a source of MDP, a substance that is sensed by and activates NOD2. Such activation initiates a mechanism of inhibition of PGN-mediated NF-kB activation and thus causes downmodulation of TLR-induced cytokine production. In patients with Crohn disease bearing NOD2 mutations (right inset), the NOD2 modulation is impaired, and hence the innate immune "thermostat" of the intestine is set at a higher level of proinflammatory cytokine production. This, together with a tendency of T cells to react to mucosal antigens, leads to inflammation and disease. An additional factor relates to the function of $\alpha$-defensins, antibacterial peptides produced by Paneth cells at the base of crypts in the terminal ileum (middle inset). Crohn disease patients with impaired NOD2 function manifest reduced $\alpha$-defensin production in part because NOD2 in Paneth cells is an inducer of $\alpha$-defensin production. Thus, it is possible that impaired NOD2 function also leads to increased bacterial density in the crypts of the terminal ileum and thus greater stimulation of a mucosal immune system already set at a higher level of function.

Microbial components trigger IBD in mice with immune defects. Interestingly, mice with NOD2 deficiency do not develop spontaneous colitis despite having a normal population of commensal gut microflora. Inflammation does ensue, however, when these mice are exposed to a specific antigen that is recognized by a critical mass of their $\mathrm{T}$ cells. This was initially demonstrated by the fact that APCs from NOD2-deficient mice display greatly increased IL-12 production and induced IFN- $\gamma$ responses in vitro when stimulated by a recombinant E. coli-expressing OVA peptide (ECOVA) and mixed with $T$ cells bearing a transgenic OVA peptide cognate TCR (29). The increased response was attributable to the fact that, in the absence of NOD2-mediated inhibition, APCs that are processing and presenting E. coli antigens (including ECOVA) mount excessive IL-12 responses to the PGN associated with the E. coli and thus greatly augment the IFN- $\gamma$ response of the T cells capable of responding to the presented antigens (again, including OVA peptide). Based on these findings, T cells bearing a TCR-recognizing OVA peptide were adoptively transferred into NOD2-deficient and NOD2-intact mice, and the animals were then challenged with ECOVA. As might be expected from the in vitro studies described above, it was found that the NOD2-deficient mice, but not NOD2-intact mice, developed severe IL-12-driven colitis. Furthermore, NOD2-deficient mice that were also TLR2-deficient did not develop colitis, indicating that the colitis was in fact due to an unmodulated TLR2 response. These studies thus suggest that NOD2 deficiency predisposes to mucosal inflammation but only in the presence of a second defect, an excessive immune response to a gut microflora antigen (Figure 1).

The finding that a single antigen, in this case OVA peptide expressed by ECOVA can cause colitis in the presence of NOD2 deficiency replicated previous studies showing that single antigens (or single organisms) that are normal components of the intestinal flora can cause colitis, provided that $\mathrm{T}$ cells are present to respond to these antigens in the context of a dysregulated mucosal immune system. It has been shown, for instance, that immunodeficient RAG2-KO or SCID mice replete with naive T cell populations bearing a TCR transgene that recognizes the OVA peptide also develop colitis if challenged with ECOVA $(30,31)$. Similarly, SCID mice replete with $\mathrm{T}$ cell lines specific for flagellin develop colitis, in this case because flagellin is a normal compo- 
nent of the bacterial flora (32). In both of these examples, dysregulated mucosal immune responses are involved because the repleting cell populations lack Tregs that ordinarily moderate responses to bacterial antigens. It should be noted, however, that while single antigens are sufficient to cause colitis in experimental animals, it does not necessarily follow that single antigens, or indeed a single organism, causes IBD.

A more general examination of the role of specific antigens in the pathogenesis of mucosal inflammation comes from the extensive studies of the role of antigens in the gut microflora in the development of cecal colitis that develops spontaneously in a substrain of $\mathrm{C} 3 \mathrm{H} / \mathrm{HeJ}$ mice, called $\mathrm{C} 3 \mathrm{H} / \mathrm{HeJBir}$ mice (33). These mice have a defect in TLR4 signaling and therefore do not respond to a major TLR ligand, LPS; thus the reason they develop colitis may be related to the loss of an inhibitory effect that LPS exerts on other TLR ligands. In the studies of these mice relevant here, it was shown that while the mice mount a B cell (antibody) response to a very considerable number of gut antigens, the overall response is restricted in that most of the antibodies are directed toward antigens arising from only Enterobacteriaceae and Enterococcus species, which make up less than 1 percent of the bacterial flora (34). The mice also mount a $\mathrm{T}$ cell response to gut antigens, but it is unclear if this response is as restricted as the $\mathrm{B}$ cell response since in this case a variety of organisms elicit response, including those present in great numbers such as Eubacteria (35). Overall, it is clear that, while the immune response may be somewhat restricted in inflammation in this model (and by extension, in other models and in human disease), many antigens are involved. One possible explanation for these findings is that the response to gut microflora during inflammation is characterized by "epitope spreading," i.e., a phenomenon frequently observed in autoimmune states whereby a primary autoimmune response to a dominant autoantigen is subsequently accompanied by a response to many autoantigens because the inflammatory state enables secondary abrogation of tolerance to many secondary autoantigens (36). Evidence in support of this possibility and the existence of a dominant autoantigen came from studies using a technique known as serological expression cloning, in which it was shown that a relatively high proportion (about $25 \%$ ) of the antigens reacting with antibodies in sera from $\mathrm{C} 3 \mathrm{H} / \mathrm{HeJBir}$ mice (and other mouse models of inflammation) were in fact flagellins (32). This, along with the facts that $\mathrm{C} 3 \mathrm{H} / \mathrm{HeJBir} \mathrm{T}$ cells reacting with flagellin can cause colitis when transferred to immunodeficient mice (as noted above) and that anti-flagellin antibodies are frequently present in Crohn disease patients (but not ulcerative colitis patients), led to the view that flagellin was a dominant antigen in both experimental colitis and in patients with Crohn disease $(32,37)$. However, the data at hand is equally consistent with the views that many bacterial antigens elicit responses from a dysregulated immune system and that the anti-flagellin response seems dominant only because it is a powerful antigen that rapidly assumes prominence in an autoreactive milieu. Indeed, this interpretation is favored by recent evidence that $\mathrm{C} 3 \mathrm{H} / \mathrm{HeJBir}$ mice bear a genetic locus on chromosome 3 harboring an as yet unidentified gene that gives rise to increased $\mathrm{T}$ cell responses to colonic bacterial antigens as well as flagellin (38).

To summarize, the linkage of CARD15/NOD2 mutations to excessive innate immune responses toward components of gut microflora represents the first time that IBD (or at least, Crohn disease) can be shown to result from mucosal hyperreactivity to normal commensal components. Furthermore, from studies that show that this molecular defect in innate immunity leads to the facilitation of adaptive immune responses to one or many individual antigens in the microflora we can infer a basis for the observation that IBD is characterized by antibodies and $\mathrm{T}$ cell responses directed to components of the microflora. Whether a similar mechanism involving other innate immune elements underlies the pathogenesis of disease in patients with IBD who do not have CARD15 mutations will be a fruitful area of future research.

\section{The case for the presence of abnormal microflora}

The second major hypothesis regarding the pathogenesis of IBD views the disease as resulting from a problem with the microflora, which induces a pathologic response from a normal mucosal immune system. Two types of evidence support this second hypothesis. One body of data suggests that IBD is associated with pathologic organisms that establish a type of low-grade infection of the mucosa and in doing so evoke the inflammatory response that characterizes the disease. Another body of data suggests that IBD patients have a defective epithelial barrier that enables the proliferation of nonpathologic organisms in close juxtaposition to elements of the mucosal immune system, again evoking the inflammatory response that characterizes the disease.

Differences in gut microflora in IBD. To examine the first body of evidence, we will consider the results of several recent studies of the bacterial microflora associated with the mucosa (rather than with the fecal microflora), since these are the bacteria likely to cause infection. Using conventional culture techniques in one such study, Swidsinski et al. demonstrated higher amounts of mucosa-associated bacteria (i.e., bacteria in the mucus layer and at the epithelial surface) in biopsy tissue obtained from patients with IBD compared with tissue obtained from control subjects (39). However, there was no evidence of bacterial penetration into the lamina propria, and noninflamed areas were actually associated with a higher amount of adherent bacteria than was inflamed mucosa. In another such study, Darfeuille-Michaud et al. also reported increased numbers of mucosa-associated bacteria in IBD (40). In this case, a pathogen-like invasive E. coli (as defined by in vitro studies) was associated with the mucosa of $20 \%-40 \%$ of ileal biopsy specimens from Crohn disease patients as compared with the mucosa of $6 \%$ of specimens from controls. In contrast, about $4 \%$ of colonic specimens from both Crohn disease patients and from control subjects harbored invasive bacteria, compared with $12 \%$ of specimens from ulcerative colitis patients. The significance of these findings, however, was thrown into question by a more recent study by Martin et al., who reported that much higher rates of mucosa-adherent bacteria can be found in the majority of Crohn patients and in substantial numbers of controls (80\% in Crohn patients and $40 \%$ in controls) and that the adherent bacteria were not limited to the ileum (41). In addition, the presence of adherent bacteria was not disease specific, in that a similar percentage occurred in colon cancer patients and Crohn disease patients. Finally, the nature of the mucosa-adherent bacteria was different from that described in the earlier study, in that only about $50 \%-75 \%$ of isolates could be classified as E. coli.

Complementary studies using molecular techniques to identify specific bacterial groups have also been applied to the study of the bacterial microflora in IBD (42-44). These studies do not support the presence of a specific, pathogenic organism in IBD, in that profiles of mucosa-associated bacterial ribosomal DNA showed no difference between inflamed and uninvolved areas. In addition, 
each individual patient displayed a distinct bacterial profile that was not characteristic of IBD patients generally. These studies were also inconsistent in that one group reported an overrepresentation of the phylum Bacteriodetes in Crohn disease and ulcerative colitis tissue, while another group showed no specific bacterial phylum associated with diseased or control tissue. Overall, any reported differences in phylogenetic groups between patients and controls were not sufficiently reproducible to justify the conclusion that organisms of any one group was associated with IBD (43).

While the data above offer little support for the idea that a mucosal infection by a specific organism is the cause of either major form of IBD, they do suggest that some changes in the microflora are present in this group of diseases that are not a secondary effect of the inflammation. This follows from the fact that the changes at not specifically associated with lesion sites. It is nevertheless possible that these changes are secondary to abnormal mucosal immune responses. The latter is supported by recent work showing that the ability of mice to mount innate (NKT cell) responses affects the composition of gut commensal microflora (45).

Investigations into altered epithelial bacterial defenses in IBD. We shall now turn to the second body of evidence supporting the view that IBD is due to the gut microflora, namely the evidence that IBD is characterized by a defective epithelial barrier that allows excessive interaction of a normal mucosal immune system with normal microflora. The relevant data here is the recent finding that Crohn disease patients exhibit decreased secretion of cationic peptides with antibacterial properties known as defensins. These defensins include either $\alpha$-defensins produced by secretory Paneth cells located at the base of the small intestinal crypts or $\beta$-defensins produced by colonic epithelial cells. It was shown that patients with Crohn disease (cases not associated with NOD2 mutations) exhibit an approximately $50 \%$ decrease in expression levels of human $\alpha$-defensin 5 (HD5) compared with controls, whereas other Paneth cell antibacterial factors were present at normal levels or even increased levels, suggesting a specific and primary defect in $\alpha$-defensin production (46). HD6 levels were similarly reduced. It should be noted, however, that HD5 and HD6 secretion was reduced in ileal specimens regardless of disease severity as well as in patient specimens without evidence of inflammation. While this finding was taken as another indication that the defect in $\alpha$-defensin production is a primary defect, it can also be interpreted as evidence that the defect is not sufficient for the occurrence of disease. Finally, bacterial-killing capacity assays of ileal biopsy extracts from patients and control subjects revealed that Crohn disease patient extracts had somewhat reduced, but still considerable, killing capacity, thus suggesting that the decrease in $\alpha$-defensin levels may be physiologically significant (46). In a parallel study of $\beta$-defensins, it was shown that ulcerative colitis but not Crohn disease patients manifest increased levels of $\beta$-defensins 2 and 3 , and it was suggested that in Crohn disease there is a lack of $\beta$-defensin induction and thus a relative deficiency of this defensin (47). Later, this abnormality was shown to have a genetic basis, in that a polymorphism in the defensin gene cluster linked with low $\beta$-defensin copy number was associated with colonic Crohn disease (48).

The above data relating to the $\alpha$-defensin production defect in Crohn disease gained additional credibility when it became apparent that NOD2 might be involved in $\alpha$-defensin expression. First, as mentioned earlier, NOD2 is expressed in Paneth cells, and in studies of epithelial cell lines it has been shown that NOD2 protects epithelial cells from bacterial infection (49). Second and more intriguingly, mutations in NOD2 affect $\alpha$-defensin production in patients with Crohn disease: patients with one form of mutation (the frameshift mutation at Leu1007) manifested a further decrease in HD5 levels than that seen in patients without NOD2 mutations, whereas patients with the two other types of mutations (Arg702Trp and Gly908Arg) manifested levels equal to those found in patients without NOD2 mutations. In addition, even in the patients with the frameshift mutation, no significant reduction in HD6 was seen compared with other Crohn disease patients. Thus, not all NOD2 mutations are associated with effects on $\alpha$-defensin production, and even the frameshift mutation does not affect all $\alpha$-defensins. Finally, the relation of NOD2 to defensin production was corroborated by studies of NOD2-deficient mice conducted by Kobayashi et al. (50). These investigators showed that NOD2-deficient mice manifested increased susceptibility to Listeria monocytogenes infection when $L$. monocytogenes was administered orally but not when administered parenterally. This finding was associated with decreased levels of two specific types of mouse cryptin mRNA (defensin homologs) in Paneth cells. However, this finding could not be tied to intestinal inflammation because NOD2-deficient mice orally infected with $L$. monocytogenes developed liver disease, not intestinal disease.

\section{Contribution of an abnormal epithelial barrier to disease pathogenesis}

In assessing the significance of a defect in $\alpha$-defensin production in the causation of Crohn disease, it is important to understand that a gross disruption of epithelial barrier function can itself be a cause of gut inflammation. This was shown definitively by Hermiston and Gordon, who created a chimeric mouse expressing a dominant-negative $\mathrm{N}$-cadherin transgene in epithelium, which leads to leaky tight junctions between cells (17). Strikingly, severe inflammation developed in the lamina propria subjacent to the epithelial cells expressing the gene but not subjacent to groups of epithelial cells not expressing the gene. It does not follow from this study, however, that lesser disruptions in barrier function lead to mucosal inflammation. This is apparent from the fact that, in the normal individual, the microflora have extensive contact with the mucosal immune system without necessarily inducing inflammation. First, it has been shown that lamina propria dendritic cells can insinuate dendritic processes between epithelial cells to sample luminal antigens $(51,52)$. Second, there is now good evidence that introduction of a commensal organism into the gastrointestinal tract of germ-free mice is followed by its unimpeded entry into the Peyer patches and mesenteric lymph nodes (53). IgA responses engendered by such entry may limit further entry but is not likely to eliminate it completely, as it has been shown that IgA-coated organisms are preferentially taken up and transported to the lamina propria by $\mathrm{M}$ cells overlying the Peyer patches (54). Third and finally, it has recently been demonstrated that so-called probiotic bacteria (bacteria with antiinflammatory properties) induce the expansion of Tregs when introduced into the rectum of mice, again showing that at least some luminal organisms make contact with the mucosal immune system (55). This observation makes it clear that the microflora have limited but constant interaction with the mucosal immune system and raise the question of why these interactions do not lead to inflammation as does the gross entry of organisms when there is a major loss of tight junction function. The answer to this question is not known, but given the role of the innate immune system in the induction of gut inflammation dis- 


\section{Table 1}

Selected examples of clinical experience with emerging IBD therapies

\begin{tabular}{|c|c|c|c|}
\hline Agent & $\begin{array}{c}\text { Target } \\
\text { or action }\end{array}$ & $\begin{array}{l}\text { Reported } \\
\text { efficacy in: }\end{array}$ & Reference \\
\hline $\begin{array}{l}\text { Anti-IL-12p40/ } \\
\text { ABT874 }\end{array}$ & IL-12/IL-23 & Active CD & 10 \\
\hline Anti-IL-6 receptor & IL-6 & Active CD & 69 \\
\hline Fontolizumab & IFN- $\gamma$ & Active CD & 68 \\
\hline Visilizumab & $\begin{array}{l}\text { CD3/apoptosis- } \\
\text { activated T cells }\end{array}$ & Active UC & 73 \\
\hline Basiliximab & IL-2 receptor & Active UC & 79 \\
\hline Daclizumab & IL-2 receptor & $\begin{array}{c}\text { No effect } \\
\text { in active UC }\end{array}$ & 80 \\
\hline Natalizumab & $\alpha_{4}$ integrins & Active CD & 67 \\
\hline MLN02 & $\alpha_{4} \beta_{7}$ integrin & Active UC & 64 \\
\hline Alicaforsen & $\begin{array}{c}\text { ICAM-1 } \\
\text { (antisense } \\
\text { oligonucleotide) }\end{array}$ & $\begin{array}{l}\text { Active UC, } \\
\text { maintenance } \\
\text { of remission }\end{array}$ & 81 \\
\hline GM-CSF & Innate immune system & Active CD & 77 \\
\hline EGF & Epithelium & Active UC & 82 \\
\hline $\begin{array}{l}\text { Porcine } \\
\text { whipworm } \\
\text { oocysts }\end{array}$ & Innate immune system & Active UC & 83 \\
\hline
\end{tabular}

$\mathrm{CD}$, Crohn disease; UC, ulcerative colitis.

cussed above, it seems likely that limited incursions of commensal bacteria do not provide sufficient stimulus via the TLR system (or any other innate signaling system) to support inflammation. On the other hand, the entry of large numbers of normal commensal organisms, as in the study of mice bearing a dominant-negative $N$-cadherin gene (17), can clearly induce an immune response sufficiently robust to initiate an inflammation.

This brings us back to the possibility that a disruption of $\alpha$-defensin production and any consequent quantitative or qualitative change in the bacteria present in the intestinal crypts leads to excessive exposure of the mucosal immune system to bacteria and to the induction of disease in the absence of an immune system defect. This is not likely to be the case for several reasons. First, as we have seen, the mucosal immune system is routinely in contact with the luminal flora, and it is not at all clear that the additional bacterial load in the ileal crypts would necessarily tip the balance in favor of inflammation unless this led to a tangible invasion of the lamina propria (prior to the onset of inflammation), for which there is little evidence. Second, and by far most important, in animal models that lack Paneth cell function, one does not see the spontaneous development of gut inflammation (56). The most instructive model of this sort is mice with a deletion of the gene encoding the cystic fibrosis transmembrane conductance regulator (CFTR), which have cystic fibrosis and develop inspissation of mucus in intestinal crypts due to defects in fluid secretion (57). Such mice develop bacterial overgrowth of the gut lumen associated with blocked intestinal crypts and trapped Paneth cell products that cannot reach the luminal flora. However, neither these mice nor patients with cystic fibrosis (who also develop bacterial overgrowth) are subject to Crohn disease-like inflammation.

This lack of support for the view that an $\alpha$-defensin defect alone can be a cause of Crohn disease does not mean that epithelial barrier function defects have no role in the pathogenesis of this disease.
The fact that an $\alpha$-defensin abnormality would lead to higher bacterial concentrations in the ileal intestinal crypts could be a contributing factor to the onset of inflammation in an area of the bowel where the bacterial level is relatively low. This would explain the observation that NOD2 mutations appear to be more frequent in Crohn disease patients with ileal involvement, since one of the NOD2 mutations is associated with $\alpha$-defensin abnormalities. On the other hand, the vastly higher concentration of bacteria in the colon may make a defensin defect in this region superfluous. Finally, it should be noted that genetic studies and observations made in murine models of inflammation suggest that factors that affect epithelial cell barrier and that seem to contribute to disease pathogenesis by bringing the microflora into a closer juxtaposition with a disordered mucosal immune system act more profoundly than do $\alpha$-defensin defects (58-60). An intriguing example of this is the association with Crohn disease of functional genetic defects in cell membrane organic anion transporter 1 and 2 (OCTN1 and -2), which further increase the risk of disease when they coexist with a NOD2 mutation (61). The OCTN defect (expressed in mucosal epithelium, macrophages, and $\mathrm{T}$ cells) could lead to altered processing of bacteria and their metabolites and result in impaired epithelial function, thus exposing a normal or disordered immune system to excess bacterial exposure.

\section{Conclusion}

The discussion above leads to the conclusion that the fundamental basis of IBD is the presence of one or more genetically determined defects that result in a mucosal immune system that overreacts to normal constituents of the mucosal microflora. These defects are abetted by genetically determined alterations in gut epithelial barrier function that enhance exposure of the mucosal immune system to microflora components. It is important to understand, however, that regardless of the particular defects, the disease process is inevitably channeled into a final common immunopathologic pathway comprised of either a Th1-type $\mathrm{T}$ cell-mediated inflammation (Crohn disease) or a Th2-type T cell-mediated inflammation (ulcerative colitis). This implies that, regardless of the nature of the fundamental defects present, one could potentially treat IBD with therapy that addresses an essential element of the final common pathway.

Within this context, existing conventional treatments such as corticosteroids, mesalamine, and immunosuppressants aim broadly to block downstream inflammatory events such as the secretion of cytokines and the elaboration of immunocytes and neutrophils, regardless of the nature of the underlying $T$ cell response that generated these events. These agents have sustained treatment of IBD for many years despite shortcomings and toxicities. Newer therapies such as antibodies against TNF- $\alpha$ and $\alpha$-integrin molecules target the mechanisms of inflammation more narrowly by eliminating a specific major inflammatory cytokine or by disrupting accumulation of cells at areas of inflammation. Both strategies have been successful in subsets of IBD patients but have also been associated with significant complications, including fatal infections (62-67). Emerging treatments (Table 1) are further evaluating the hierarchy of the inflammatory cytokine effect by targeting IL-12/IL-23 (10), IFN- $\gamma$ (68), or IL-6 (69) or by restoring IL-10 levels (70). AntiIL-12p40, an antibody that targets IL-12 and IL-23, the master cytokines underlying the Th1 response, has been shown in preliminary studies mentioned above to have striking efficacy in Crohn disease (10). The possible use of a related antibody, anti-IL-23p19, which targets IL-23 (and not IL-12), has been suggested as a poten- 
tially useful treatment because of recent reports that experimental colitis is more dependent on IL-23 and IL-17 than on IL-12 and IFN- $\gamma$, but this remains to be tested by a clinical trial. Other strategies aim to eliminate effector cells by leukocytapheresis $(71,72)$, administration of anti-CD3 antibodies (73), and autologous hematopoietic stem cell transplant (74). Other approaches to the treatment of IBD currently under investigation are aimed at restoring the lack of immunoregulation, (e.g., extracorporeal photophoresis [ref. 75], adipose stem cell infusion [ref. 76], and administration of probiotics), enhancing innate immune function (via use of GM-CSF [ref. 77]), or activating the innate immune system by administration of microbe-derived agents, with the aim of inducing counterregulatory immune responses to quell established inflammation (78). These treatments should result in reduction of the inflammatory mediators that cause tissue damage, maintenance of the active inflammation, and exacerbation of dysfunction of the epithelial barrier, ultimately leading to control of the second- ary effects without correcting the primary genetic susceptibility factors that lead to the development of the IBD in the first place. The challenges facing those involved in the development of new therapies for IBD, will include the identification of new molecular defects such as NOD2 mutations, as well as, the elucidation of the functional impact of these defects on mucosal immune responses and epithelial cell barrier function. In addition, these challenges will include the continued study of the intestinal microflora so as to identify the specific components that enhance or antagonize mucosal immune responsiveness and nonresponsiveness. Needless to say, such molecularly grounded approaches hold the promise of more effective therapies with fewer side effects.

Address correspondence to: Warren Strober, National Institutes of Health, Bldg. 10, CRC, Room 5-3940, Bethesda, Maryland 20892, USA. Phone: (301) 496-6810; Fax: (301) 402-2240; E-mail: wstrober@niaid.nih.gov.
1. Podolsky, D. 2002. Inflammatory bowel disease. N. Engl. J. Med. 347:417-429.

2. Fuss, I., et al. 2004. Nonclassical CD1d-restricted NK T cells that produce IL-13 characterize an atypical Th2 response in ulcerative colitis. J. Clin. Invest. 113:1490-1497. doi:10.1172/JCI200419836.

3. McLeod, R. 2003. Surgery for inflammatory bowel diseases. Dig. Dis. 21:168-179.

4. Feagan, B. 2003. Maintenance therapy for inflammatory bowel disease. Am. J. Gastroenterol. 98(Suppl. 12):S6-S17.

5. Sandborn, W. 2006. Treatment of ulcerative colitis with oral mesalamine: advances in drug formulation, efficacy expectations and dose response, compliance, and chemoprevention. Rev. Gastroenterol. Disord. 6:97-105.

6. Siegel, C., and Sands, B. 2005. Practical management of inflammatory bowel disease patients taking immunomodulators. Aliment. Pharmacol. Ther. 22:1-16.

7. Targan, S. 2006. Current limitations of IBD treatment: where do we go from here? Ann. N. Y. Acad. Sci. 1072:1-8

8. Fuss, I., et al. 2006. Both IL-12p70 and IL-23 are synthesized during active Crohn's disease and are down-regulated by treatment with anti-IL-12 p40 monoclonal antibody. Inflamm. Bowel Dis. 12:9-15.

9. Yen, D., et al. 2006. IL-23 is essential for T cellmediated colitis and promotes inflammation via IL-17 and IL-6. J. Clin. Invest. 116:1310-1316. doi:10.1172/JCI21404.

10. Mannon, P., et al. 2004. Anti-interleukin-12 antibody for active Crohn's disease. N. Engl. J. Med. 351:2069-2079.

11. Schappi, M., et al. 2003. The nature of colitis in chronic granulomatous disease. J. Pediatr. Gastroenterol. Nutr. 36:623-631.

12. Mannon, P., et al. 2006. Excess IL-12 but not IL-23 accompanies the inflammatory bowel disease associated with common variable immunodeficiency. Gastroenterology. 131:748-756.

13. Hussain, N., et al. 2006. Intestinal disease in Hermansky-Pudlak syndrome: occurrence of colitis and distribution with genotype. Clin. Gastroenterol. Hepatol. 4:73-80.

14. Phan, G., et al. 2003. Cancer regression and autoimmunity induced by cytotoxic $\mathrm{T}$ lymphocyteassociated antigen 4 blockade in patients with metastatic melanoma. Proc. Natl. Acad. Sci. U. S. A. 100:8372-8377.

15. Duchmann, R., Herman, E., Mayet, W., Ewe, K., and Meyer zum Buschenfelde, K.H. 1995. Tolerance exists towards resident intestinal flora but is broken in active inflammatory bowel disease (IBD). Clin. Exp. Immunol. 102:448-455.
16. Strober, W., Fuss, I., and Blumberg, R. 2002. The immunology of mucosal models of inflammation. Annu. Rev. Immunol. 20:495-549.

17. Hermiston, M., and Gordon, J. 1995. Inflammatory bowel disease and adenomas in mice expressing a dominant negative $\mathrm{N}$-cadherin. Science. 270:1203-1207.

18. Inohara, N., and Nunez, G. 2003. NODS: intracellular proteins involved in inflammation and apoptosis. Nat. Rev. Immunol. 3:371-382.

19. Strober, W., Murray, P., Kitani, A., and Watanabe, T. 2006. Signalling pathways and molecular interactions of NOD1 and NOD2. Nat. Rev. Immunol. 6:9-20.

20. Kobayashi, M., et al. 2003. Toll-like receptordependent production of IL-12p40 causes chronic enterocolitis in myeloid cell-specific Stat3-deficient mice. J. Clin. Invest. 111:1297-1308. doi:10.1172/ JCI200317085.

21. Rakoff-Nahoum, S., Hao, L., and Medzhitov, R. 2006. Role of toll-like receptors in spontaneous commensal-dependent colitis. Immunity. 25:319-329.

22. Hugot, J., et al. 2001. Association of NOD2 leucinerich repeat variants with susceptibility to Crohn's disease. Nature. 411:599-603.

23. Ogura, Y., et al. 2001. A frameshift mutation in NOD2 associated with susceptibility to Crohn's disease. Nature. 411:603-606.

24. Watanabe, T., Kitani, A., Murray, P., and Strober, W. 2004. NOD2 is a negative regulator of Toll-like receptor2-mediated T helper type 1 responses. Nat. Immunol. 5:800-808.

25. Kobayashi, K., et al. 2002. RICK/Rip2/CARDIAK mediates signalling for receptors of the innate and adaptive immune systems. Nature. 416:194-199.

26. Abbott, D., Wilkins, A., Asara, J., and Cantley, L. 2004. The Crohn's disease protein, NOD2, requires RIP2 in order to induce ubiquitinylation of a novel site on NEMO. Curr. Biol. 14:2217-2227.

27. Uehara, A., et al. 2005. Muramyldipeptide and diaminopimelic acid-containing desmuramylpeptides in combination with chemically synthesized Toll-like receptor agonists synergistically induced production of interleukin-8 in a NOD2 and NOD1-dependent manner, respectively, in human monocytic cells in culture. Cell. Microbiol. 7:53-61.

28. Zelinkova, Z., et al. 2005. Functional consequences of NOD2 deficiency in Crohn's disease patients peripheral blood blood monocytes derived dendritic cells. Gastroenterology. 128:A510.

29. Watanabe, T., et al. 2006. Nucleotide binding oligomerization domain 2 deficiency leads to dysregulated TLR2 signaling and induction of antigen-specific colitis. Immunity. 25:473-485.

30. Iqbal, N., et al. 2002. T helper 1 and Thelper 2 cells are pathogenic in an antigen specific model of colitis. J. Exp. Med. 195:71-84.

31. Yoshida, M., et al. 2001. CD4 T cells monospecific to ovalbumin produced by Eschericia coli can induce colitis upon transfer to BALB/c and SCID mice. Int. Immunol. 13:1561-1570.

32. Lodes, M., et al. 2004. Bacterial flagellin is a dominant antigen in Crohn's disease. J. Clin. Invest. 113:1296-1306. doi:10.1172/JCI200420295.

33. Elson, C., and Cong, Y.2002. Understanding immunemicrobial homeostasis in intestine. Immunol. Res. 26:87-94.

34. Brandwein, S., et al. 1997. Spontaneous colitic $\mathrm{C} 3 \mathrm{H} / \mathrm{HeJBir}$ mice demonstrate selective antibody reactivity to antigens of the enteric bacterial flora. J. Immunol. 159:44-52.

35. Cong, Y., et al. 1998. CD4+ T cells reactive to enteric bacterial antigens in spontaneously colitic $\mathrm{C} 3 \mathrm{H} / \mathrm{HeJBir}$ mice: increased T helper cell type 1 response and ability to transfer disease. J. Exp. Med. 187:855-864.

36. Robinson, W., et al. 2003. Protein microarrays guide tolerizing DNA vaccine treatment of autoimmune encephalitis. Nat. Biotechnol. 21:1017-1019.

37. Targan, S., et al. 2005. Antibodies to CBirt flagellin define a unique response that is associated independently with complicated Crohn's disease. Gastroenterology. 128:2020-2028.

38. Beckwith, J., Cong, Y., Sundberg, J., Elson, C., and Leiter, E. 2005. Cdcs1, a major colitogenic locus in mice, regulates innate and adaptive immune responses to enteric bacterial antigens. Gastroenterology. 129:1473-1484.

39. Swidsinski, A., et al. 2002. Mucosal flora in inflammatory bowel disease. Gastroenterology. 122:44-54.

40. Darfeuille-Michaud, A., et al. 1998. Presence of adherent Escherichia coli strains in ileal mucosa of patients with Crohns' disease. Gastroenterology. 115:1405-1413.

41. Martin, H., et al. 2004. Enhanced Eschericia coli adherence and invasion in Crohn's disease and colon cancer. Gastroenterology. 127:80-93.

42. Bibiloni, R., Mangold, M., Madsen, K., Fedorak, R., and Tannock, G. 2006. The bacteriology of biopsies differs between newly diagnosed, untreated Crohn's disease and ulcerative colitis patients. J. Med. Microbiol. 55:1141-1149.

43. Prindiville, T., Cantrell, M., and Wilson, K. 2004. Ribosomal DNA sequence analysis of mucosaassociated bacteria in Crohn's disease. Inflamm. Bowel Dis. 10:824-833.

44. Seksik, P., et al. 2006. Review article: the role of bacteria in onset and perpetuation of inflammatory bowel disease Aliment. Pharmacol. Ther. 24:11-18.

45. Niewenhuis, E. 2006. CD1d-dependent regulation 
of bacterial colonization in the intestine. Gastroenterology. 130:A61.

46. Wehkamp, J., et al. 2005. Reduced Paneth cell alpha-defensins in ileal Crohn's disease. Proc. Natl. Acad. Sci. U. S. A. 102:18129-18134.

47. Wehkamp, J., et al. 2003. Inducible and constitutive beta-defensins are differentially expressed in Crohn's disease and ulcerative colitis. Inflamm. Bowel Dis. 9:215-223.

48. Fellerman, K., et al. 2006. A chromosome 8 genecluster polymorphism with low human beta-defen$\sin 2$ gene copy number predisposes to Crohn's disease in the colon. Am. J. Hum. Genet. 79:439-448.

49. Hisamatsu, T., et al. 2003. CARD15/NOD2 functions as an antibacterial factor in human epithelial cells. Gastroenterology. 124:1145-1149.

50. Kobayashi, K.S., et al. 2005. Nod2-dependent regulation of innate and adaptive immunity in the intestinal tract. Science. 307:731-734.

51. Niess, J., et al. 2005. CX3CR1-mediated dendritic cell access to the intestinal lumen and bacterial clearance. Science. 307:254-258

52. Rescigno, M., et al. 2001. Dendritic cells express tight junction proteins and penetrate gut epithelial monolayers to sample bacteria. Nat. Immunol. 2:361-367.

53. Macpherson, A., and Uhr, T. 2004. Induction of protective IgA by intestinal dendritic cells carrying commensal bacteria. Science. 303:1662-1665.

54. Mantis, N., et al. 2002. Selective adherence of IgA to murine Peyer's patch M cells: evidence for a novel IgA receptor. J. Immunol. 169:1844-1851.

55. DiGiacinto, C., Marinaro, M., Sanchez, M., Strober, W., and Boirivant, M. 2005. Probiotics ameliorate recurrent Th1-mediated murine colitis by inducing IL-10 and IL-10-dependent TGF- $\beta$-bearing regulatory cells. J. Immunol. 174:3237-3246.

56. Wilson, C., et al. 1999. Regulation of intestinal alpha-defensin activation by the metalloproteinase matrilysin in innate host defense. Science. 286:113-117.

57. Norkina, O., Burnett, T., and Lisle, R.D. 2004. Bacterial overgrowth in the cystic fibrosis transmembrane conductance regulator null mouse small intestine. Infect. Immun. 72:6040-6049.

58. Ho, G., et al. 2006. ABC1/MDR1 gene determines susceptibility and phenotype in ulcerative colitis: discrimination of critical variants using a genewide haplotype tagging approach. Hum. Mol. Genet.
15:797-805.

59. Newman, B., et al. 2005. A risk haplotype in the Solute Carrier Family 22A4/22A5 gene cluster influences phenotypic expression of Crohn's disease. Gastroenterology. 128:260-269.

60. Panwala, C., Jones, J., and Viney, J. 1998. A novel model of inflammatory bowel disease: mice deficient for the multiple drug resistance gene, mdr1a, spontaneously develop colitis. J. Immunol. 161:5733-5744.

61. Peltekova, V., et al. 2004. Functional variants of OCTN cation transporter genes are associated with Crohn disease. Nat. Genet. 36:471-475.

62. Adelman, B., Sandrock, A., and Panzara, M. 2005. Natalizumab and progressive multifocal leukoencephalopathy. N. Engl. J. Med. 353:432-433.

63. Blonski, W., and Lichtenstein, G. 2006. Complications of biological therapy for inflammatory bowel diseases. Curr. Opin. Gastroenterol. 22:30-43.

64. Feagan, B., et al. 2005. Treatment of ulcerative colitis with a humanized antibody to the alpha4beta7 integrin. N. Engl. J. Med. 352:2499-2507.

65. Hanauer, S., et al. 2002. Maintenance infliximab for Crohn's disease: the ACCENT I randomised trial. Lancet. 359:1541-1549.

66. Rutgeerts, P., et al. 2005. Infliximab for induction and maintenance therapy for ulcerative colitis. N. Engl. J. Med. 353:2462-2476.

67. Sandborn, W., et al. 2005. Natalizumab induction and maintenance therapy for Crohn's disease. N. Engl. J. Med. 353:1912-1925.

68. Hommes, D., et al. 2006. Fontolizumab, a humanised anti-interferon gamma antibody, demonstrates safety and clinical activity in patients with moderate to severe Crohn's disease. Gut. 55:1131-1137.

69. Ito, H., et al. 2004. A pilot randomized trial of a human anti-interleukin- 6 receptor monoclonal antibody in active Crohn's disease. Gastroenterology. 126:989-996

70. Braat, H., et al. 2006. A phase I trial with transgenic bacteria expressing interleukin-10 in Crohn's disease. Clin. Gastroenterol. Hepatol. 4:754-759.

71. Nishioka, C., et al. 2005. Leukocytapheresis therapy for steroid-naive patients with active ulcerative colitis: its clinical efficacy and adverse effects compared with those of conventional steroid therapy. J. Gastroenterol. Hepatol. 20:1567-1571.

72. Sands, B., et al. 2006. Pilot feasibility studies of leukocytapheresis with the Adacolumn Apheresis
System in patients with active ulcerative colitis or Crohn disease. J. Clin. Gastroenterol. 40:482-489.

73. Targan, S., et al. 2005. A phase I-II Study: multiple dose levels of visilizumab are well tolerated and produce rapid and sustained improvement in ulcerative colitis patients refractory to treatment with iv steroids (IVSR-UC). Gastroenterology. 128:A75.

74. Oyama, Y., et al. 2005. Autologous hematopoietic stem cell transplantation in patients with refractory Crohn's disease. Gastroenterology. 128:552-563.

75. Reinisch, W., et al. 2001. Extracorporeal photochemotherapy in patients with steroid-dependent Crohn's disease: a prospective pilot study. Aliment. Pharmacol. Ther. 15:1313-1322.

76. Garcia-Olmo, D., et al. 2005. A phase I clinical trial of the treatment of Crohn's fistula by adipose mesenchymal stem cell transplantation. Dis. Colon Rectum. 48:1416-1423.

77. Korzenik, J., Dieckgraefe, B., Valentine, J., Hausman, D., and Gilbert, M.J. 2005. Sargramostim for active Crohn's disease. N. Engl. J. Med. 352:2193-2201.

78. Weinstock, J. 2006. Helminths and mucosal immune modulation. Ann. N. Y. Acad. Sci. 1072:356-364.

79. Creed, T., Norman, M., and Probert, C. 2003. Basiliximab (anti-CD25) in combination with steroids may be an effective new treatment for steroidresistant ulcerative colitis. Aliment. Pharmacol. Ther. 18:65-75.

80. Van Assche, G., et al. 2006. Daclizumab, a humanized monoclonal antibody to the interleukin 2 receptor (CD25), for the treatment of moderately to severely active ulcerative colitis: a randomized, double blind, placebo controlled, dose ranging trial. Gut. 55:1568-1574.

81. Van Deventer, S., Volfova, M., and Flisiak, R. 2005. A phase 2 dose-ranging, double blind, placebo-controlled study of alicaforsen enema in subjects with acute exacerbation of mild to moderate left-sided ulcerative colitis. Gastroenterology. 128:A74.

82. Sinha, A., Nightingale, J., West, K., Berlanga-Acosta, J., and Playford, R. 2003. Epidermal growth factor enemas with oral mesalamine for mild-to-moderate left-Sided ulcerative colitis or proctitis. N. Engl. J. Med. 349:350-357.

83. Summers, R., Elliott, D., and Urban, J. 2005. Trichuris suis therapy for active ulcerative colitis: a randomized controlled trial. Gastroenterology. 128:825-832 